\title{
Blasenentleerungsstörungen oft „Folge“ von Pflegebedürftigkeit?!
}

\begin{abstract}
Dass Blasenentleerungsstörungen bei älteren Menschen, besonders bei Patienten mit fortgeschrittener Pflegebedürftigkeit und demenziellen Erkrankungen, ein häufiges Problem sind, dürfte bekannt sein. Ziel einer Studie aus Österreich war es daher, die Beziehung zwischen der Diagnose einer Blasenentleerungsstörung und den im Rahmen eines geriatrischen Assessments definierten altersassoziierten funktionellen Defiziten darzulegen.
\end{abstract}

") Oft sind die Blasenentleerungsstörungen bei älteren Menschen eine Folge von verschiedenen zugrunde liegenden körperlichen Veränderungen, funktionellen Defiziten, Komorbiditäten und Medikamentennebenwirkungen und demnach kein eigenständiges Krankheitsbild. Um den betroffenen Patienten die passende Diagnostik und Therapie zukommen zu lassen, ist es nötig, den Zusammenhang zwischen der Diagnose einer Blasenentleerungsstörung und den altersassoziierten funktionellen Defiziten zu kennen.

Die vorliegende Studie hatte das Ziel, einerseits genau diese Beziehung aufzuzeigen und anderseits die Häufigkeit (Prävalenz) von Blasenentleerungsstörungen in einem akutgeriatrischen Setting mit einer großen Stichprobe aufzudecken. Dafür wurden die Daten von 8.106 Patienten, die zwischen März 2005 und Dezember 2011 in der Abteilung für Innere Medizin und Akutgeriatrie in einem öffentlichen Krankenhaus in Österreich versorgt wurden, retrospektiv ausgewertet.
Nicht von allen Patienten lagen die vollständigen elektronischen Patientenakten vor. Daher verblieben für die Analyse die Daten von 7.487 Patienten, von denen folgende Werte oder Testergebnisse vorlagen, die zur Statistischen Auswertung herangezogen wurden:

- Alter

- Blasenentleerung(BE)-Score

- Activities of Daily Living (ADL)

- Mini Mental State Examination (MMSE)

- Geriatric Depression Scale (GDS)

- Tinetti-Mobilitätstest

- Timed up and go Test

- Gebrauch von Gehhilfen

- Handkraftmessung

- Body-Mass-Index(BMI)

- Numerical Rating Scale for Pain Intensity (NRS-Score zum Ausdruck der Schmerzintensität)

- Mini Nutritional Assessment $\left(\mathrm{MNA}^{\circledR}\right)$ Score

- Sehtest (VisionScore)

$\mathrm{Da}$ diese Tests bei allen Patienten ab einem Alter von 65 Jahren innerhalb der ersten Tage des stationären Aufent- halts routinemäßig durchgeführt werden, stand der Forschungsgruppe das beschriebene multidimensionale Assessment bzw. dessen Ergebnis verhältnismäßig unkompliziert zur Verfügung. Nach der Datenanalyse mit dem Statistikprogramm SPSS 18.0 erhielten die Forscher folgende Ergebnisse: 4.494 Patienten $(60 \%)$ wiesen Blasenentleerungsstörungen auf (davon waren $78,5 \%$ weiblich und $21,5 \%$ männlich). Von diesen 4.494 Patienten wiesen $95,8 \% \quad(n=4.304) \quad$ relevante funktionelle Defizite in drei oder mehr der durch geführten Tests auf.

Der Schweregrad der Störung korrelierte mit dem Ausmaß der meisten funktionellen Einschränkungen bzw. mit dem Alter der Betroffenen, der Hilfsbedürftigkeit in den Aktivitäten des täglichen Lebens(ADL), der benötigten Zeit für den Timed up and go Test, den Erfordernissen von Gehhilfen, der Schmerzintensität zum kognitiven (MMSE) und emotionalen (GDS) Status, zum Tinetti-Score, zur Muskelkraft, zum Ernährungszustand und zur Sehleistung. Diese Korrelationen erklären die bekannten komplexen multifaktoriellen Zusammenhänge zwischen alterstypischen körperlichen und funktionellen Veränderungen, Erkrankungen und individuellen Risikofaktoren, die kennzeichnend für geriatrische Patienten und ihre häufigsten Symptomenkomplexe, u.a. für Blasenentleerungsstörungen, sind.

Quelle

H. Talasz, M. Gosch, M. Kofler, M. Lechleitner. Blasenentleerungsstörungen bei geriatrischen Patient(inn)en. Korrelation mit altersassoziierten funktionellen Defiziten. $Z$ Gerontol Geriat (online first) DOI 10.1007/s00391-016-1097-y tragen ebenfalls zur Validität der Studie bei. Leider wurden die Daten nur von einer Abteilung eines Krankenhauses analysiert. Außerdem sind die Auswertungen bereits mindestens sechs Jahre alt. Dessen ungeachtet wird durch diese Studie nochmal deutlich aufgezeigt, dass Blasenentleerungsstörungen ein sehr häufiges (Pflege-)Problem vor allem im geriatrischen Bereich sind. Zudem belegen die Studienergebnisse, dass Blasenentleerungsstörungen sehr häufig mit (altersassoziierten) funktionellen Defiziten in Beziehung stehen. Für die (Pflege-)Praxis heißt das, dass die funktionellen Defizite bei älteren Patienten mit Blasenentleerungsstörungen beispielsweise in Bezug auf diagnostische und therapeutische Verfahren berücksichtigt werden sollten, da die Qualität der Umsetzung dieser diagnostischen und therapeutischen Verfahren durch die Blasenentleerungsstörungen beeinträchtigt sein kann.
Autorenkontakt:

Referiert und kommentiert von

Oliver Ludwig, examinierter

Gesundheits- und

Krankenpfleger und

Diplompflegepädagoge, Jena.

E-Mail: oliver_ludwig@gmx.de 